\title{
Modeling grain transportation in the system of grain processing industries
}

\author{
Abdizhapar Saparbayev ${ }^{1,2, *}$, Aiymzhan Makulova ${ }^{3, * *}$, Nesipkul Bayboltaeva ${ }^{4, * * *}$, Nagima Sarsenbieva ${ }^{5, * * * *}$, and Aliya \\ Imatayeva $^{3, \dagger}$ \\ ${ }^{1}$ Academy Kainar, 7a Satpayev Str., Almaty, 050059, Kazakhstan \\ ${ }^{2}$ International Academy of Innovative Technologies, 33 mkr. Samal-2, Almaty, 050059, Kazakhstan \\ ${ }^{3}$ University Narxoz, 55 Zhandosov Str., Almaty, 050035, Kazakhstan \\ ${ }^{4}$ NJSC “Kazakh National Agrarian University”, 8 Abay Ave., Almaty, 050010, Kazakhstan \\ ${ }^{5}$ Mukhtar Auezov South Kazakhstan University, 5 Tauke Khan Ave., Shymkent, 160012, Kazakhstan
}

\begin{abstract}
The article discusses the modeling of grain transportation in the system of grain processing industries. The question of determining the need of vehicles for the maintenance of agricultural work is of great national economic importance. It is associated with the problem of the effective use of material and cash resources, land and labor in grain farming. The resulting task is a difficult experimental task.
\end{abstract}

\section{Introduction}

To improve the efficiency of grain production, it is necessary to ensure a close connection of the grain industry with the branches engaged in the storage and processing of grain crops and the sale of their products.

Proper organization of the export of the grown grain crop plays an important role in improving the efficiency of the grain receiving enterprises (GRE) and the entire sector of the economy, as it contributes to a relative reduction in all transportation costs, improving the quality and safety of grain, reducing the cost of harvesting, improving the economic relations between the grain processing industries in the process of transportation and procurement works in a short time.

All sorts of oncoming, unnecessarily long-distance transportation of grain crops distract vehicles from transportation that is really necessary for the national economy, unreasonably increase the volume of transport and loading and unloading operations, which, in turn, causes an irrational expenditure of public funds allocated for reimbursement of expenses for the organization of transport systems. Consequently, the reduction of export costs can be achieved on the basis of rational transportation patterns by optimally linking grain farms with GRE [1].

A prerequisite for the procurement of grain is to deliver it to the GRE in the shortest possible time. At the same time, it is necessary to organize timely delivery of grain from the landlords to the GRE.

At present, a large number of economic and mathematical models in the grain processing industry have been developed [2-4], in which there are three main areas:

\footnotetext{
*e-mail: sad171@mail.ru

**e-mail: aiymzhan.makulova@narxoz.kz

***e-mail: nesipkul.bayboltaeva.53@mail.ru

****e-mail: sarsenbieva60@mail.ru

†e-mail: aliya.imatayeva@narxoz.kz
}

- development and solution of economic and mathematical problems of on-farm analysis;

- development and solution of economic and mathematical problems at the level of agro-industrial associations and individual units of the grain processing industry;

- development and solution of economic and mathematical problems of industry analysis.

At present, the tasks of the first direction are the most developed and implemented, since the information necessary for them is more accessible and reliable. The objectives of this direction include: optimization of the use of mineral and organic fertilizers; optimization of crop development plan; optimization of the production structure of the grain enterprise, etc.

The second direction, which has arisen in connection with the organization of agro-industrial associations, includes the tasks of optimizing not only the production of grain production, but also its industrial processing within the associations. The third direction is connected with the development and solution of problems of development of individual links of the grain processing industry at the level of the oblast, krai and republic. The main objective of this direction is the optimal placement and specialization of grain production by regions, as well as the optimization of purchases of grain products by farms, regions, regions and republics [2].

The main directions of improving the efficiency of transport in the grain processing industry include:

- the organization of the work of transport with the aim of the most efficient use of vehicles, labor, material and other resources in order to best meet the needs of the grain processing industries under consideration and reduce product losses; 
- the distribution and use of resources within the transport industry in such a way as to ensure the implementation of transport work with minimal economic costs;

- improvement of the vehicle fleet by rationalization of its structure taking into account the specificity of grain products.

Until now, the above-mentioned tasks of optimal use of transport systems in the grain processing industry were solved separately: either products were distributed according to a given production nomenclature without taking into account the parameters of the vehicles used, or rolling stock between the clientele, without taking into account the specificity of the product being transported, or already established links.

Proceeding from this, the problem of optimizing the use of transport systems in the grain processing industry should be considered in an integrated approach in the fieldprocurement-processing-market production complex, taking into account market relations.

In the integrated field-harvesting-processing system, on the example of the Akmola region, optimal options for attaching grain farms to grain receiving enterprises are determined on the basis of effective use of their technical ability in operations of receiving, processing, placing, forming batches of grain and the optimal scheme for linking grain receiving enterprises to processing plants taking into account the distance of transportation for the intended purpose, as well as the annual demand. These model solutions can be used to justify the capacity, throughput of grain receiving elevators and managing grain sales.

The developed models for transporting grain and grain products in the production complex "field-harvestingprocessing-market" to the interactive specialized software package "ASTYK" were introduced in the territorial departments of agriculture of the republic.

\section{Material and methods}

When optimizing the technology of grain procurement in the system "field-procurement", it is possible to determine the main parameters of the volume of procurement and the formation of optimal flows of grain transportation vehicles. These include: identifying the possible magnitude of the volume of workpieces and the proportion of grain crops, as well as determining the number of batches of grain to be formed for specific purposes, depending on the state and technological dignity.

The task of linking grain farms with GRE belongs to the class of operational planning tasks. The mathematical model of the task of linking grain farms with GRE is formulated as a multi-index transport-distributed linear programming problem of high dimensionality.

It is necessary to find in the area of permissible values such solutions, at which the work carried out by vehicles for the transportation of grain crops in the field-harvesting system is minimized.

Let us proceed to the consideration of the mathematical model of problems.
Required to minimize

$$
F(x)=\sum_{i=1}^{m} \sum_{j=1}^{n} \sum_{l=1}^{L} \sum_{t=1}^{T} R_{i l} X_{i j l t}
$$

with restrictions:

per volume of crops grown

$$
\sum_{l=1}^{L} X_{i j l t} \geq Q_{i j t}, i=\overline{1, m} ; j=\overline{1, n} ; t=\overline{1, T} ;
$$

on the volume of grain blanks of each GRE

$$
\sum_{i=1}^{m} X_{i j l t} \geq P_{l j t}, l=\overline{1, L} ; j=\overline{1, n} ; t=\overline{1, T} ;
$$

per capita capacity

$$
\alpha_{1} \leq \sum_{i=1}^{m} \sum_{j=1}^{n} \sum_{t=1}^{T} X_{i j l t}, l=\overline{1, L} ;
$$

on technical capabilities

$$
\sum_{i=1}^{m} U_{i j t} X_{l j t} \leq V_{i l t} P_{j l t}, j=\overline{1, m} ; l=\overline{1, L} ; t=\overline{1, T} ;
$$

with the natural requirement of nonnegativity of variables

$$
X_{i j l t} \geq 0, i=\overline{1, m} ; j=\overline{1, n} ; l=\overline{1, L} ; t=\overline{1, T} ;
$$

where $i$ - the index of grain farming;

$j$ - the index of the type of grain crops;

$l$ - the index of GRE;

$t$ - the index of the time subperiod;

$Q_{i j t}$ - the volume of grain crops of the $j$-th species in the $i$-th farm, which must be transported to the subperiod $t$;

$P_{j l t}-$ the volumes of preforms $l$-th GRE of $j$-th culture sub-period $t$

$\alpha_{l}-$ the the minimum amount of grain required for the smooth operation of $l$-th GRE;

$R_{i l}$ - the distance between the $i$-th grain farms and $l$-th GRE;

$U_{i l}$ - the proportion of the $j$-th culture in the $i$-th economy sub-period $t$

$V_{i l t}$ - the proportion of $j$-th culture in $l$-th GRE and subperiod $t$;

$X_{i j l t}$ - volumes of delivered grain crops of the $j$-th species from the grown $i$-th grain farming to the $l$-th in the subperiod $t$.

In the more general case, the model (1) - (6) should be supplemented with inequalities of the type (4), taking into account the grain drying capacities of the GRE. However, the mathematical content of the model will change.

When solving the problem (1) - (6) by conventional linear programming methods, considerable difficulties arise associated with the high dimensionality of the problem and the limited amount of high-speed memory of computer equipment [5]. 
The specificity of the constraint system (1) - (6) allows the use of the aggregation method: the process of solving the original problem is replaced by solving a number of problems of a much smaller dimension with the corresponding linking of the solutions obtained.

Information support of the task is based on data on the names of grain farms and GRE of the considered area, information about the capacity and throughput of GRE, distances of transportation of grain from producers to GRE, information about planned deliveries of grain from grain farms, about the availability of unloading mechanisms at GRE, etc.

\section{Results and discussion}

Experimental calculations using the model (1) - (6) were performed on real data from the Akmola regional department of agriculture and the Statistics Committee of the Ministries of the National Economy of the Republic of Kazakhstan in recent years using the developed specialized dialogue complex "ASTYK", which provides a realtime solution to the problem linking grain farms with GRE in the region. Intended for carrying out calculations on linear models of various contents of dimension and structure based on standard software, and the procedures allow: parametric generation of models, their additional calculation diagnostics, automatic setup of report generation programs, dialog management [3].

In accordance with this, the software performs:

- tabular input of information;

- control and correction of the initial information;

- calculation of the optimal linking plan;

- analysis and correction of the results;

- issue of output forms of documents.

Experimental calculations consist of three stages:

1) preparation of initial information for solving a specific variant of the problem;

2) problem solving;

3) the issuance of the results of the decision in a userfriendly form.

At the first stage, the MPS-format of data presentation was adopted for presenting initial information in modern packages for solving optimization problems of mathematical programming.

We have developed a set of programs that translate data into the MPS-format, which allows presenting input data in the form of tables, quickly and efficiently correcting the input information.

At the second stage, in order to establish the optimal variant of linking grain farms with GRE, the following tasks were solved:

- grouping of grain farms according to GRE, taking into account the existing list on the basis of contracting agreements;
- grouping of grain farms according to GRE, taking into account the shortest distance of grain delivery within the region;

- grouping of grain farms according to GRE, taking into account the shortest distance of grain delivery within the region.

The calculation was carried out in the context of 104 grain farms and 33 GRE area, where the power GRE correspond to the volume of produced grain. Minimum operating costs are obtained on the basis of a decrease in the distance of grain transportation by road from grain farms to GRE.

When evaluating the binding scheme of the studied variants, the analysis of the solution showed that the second option is considered the most optimal, i.e. linkage of grain farms for GRE, taking into account the shortest distance of grain delivery within the region.

As a result of work of farms, GRE and automobile transport in accordance with the optimal scheme of linking farms with GRE, the average distance of grain delivery to enterprises decreased against the existing one by 13.1 $\mathrm{km}$, i.e. reduced the cost of transporting 1 ton of grain by 3024 tenge in the whole area. From optimizing the linkage of grain farms with GRE, the overall economic efficiency amounted (estimated) to 709,128 thousand tenge.

At the third stage, on the basis of the decision received and the initial information, the technical and economic indicators of interest to us are calculated in the form of reports.

Experimental calculations showed the need to take into account in the model (1) - (6) still a number of conditions and restrictions, and those that are usually unknown in advance and can only be updated by a planner directly in the process of forming a plan.

In the above model (1) - (6) it is assumed that all quantities are deterministic, but research shows that these quantities cannot be considered as predetermined, since they can vary significantly under different conditions. Therefore, the deterministic model (1) - (6) does not reflect the conditions associated with the random nature of the quantities, and it becomes necessary to consider models of stochastic programming that take into account the probabilistic nature of the initial information [4-7].

Suppose that all the data of the model (1) - (6), except for the right-hand sides of inequalities (3) and (5), are known in advance. Let the value of $P_{i l t}-$ the volume of billet grain GRE - is a random variable with a given distribution law. Then problem (1) - (6) can be formulated as a one-stage stochastic problem in the form. 


$$
\begin{gathered}
Z=\sum_{i=1}^{m} \sum_{j=1}^{n} \sum_{l=1}^{L} \sum_{t=1}^{T} R_{i l} X_{i j l t}+ \\
+M\left(\sum_{j=1}^{n} \sum_{l=1}^{L} \sum_{t=1}^{T} \alpha_{j l t}\left(\max \left(0 ; \sum_{i=1}^{m} X_{i j l t}-P_{j l t}(\Theta)\right)\right)\right)+ \\
+M\left(\sum_{j=1}^{n} \sum_{l=1}^{L} \sum_{t=1}^{T} \beta_{j l t}\left(\max \left(0 ; \sum_{i=1}^{m} U_{i j t} X_{i j l t}-V_{j l t} P_{l j t}(\Theta)\right)\right)\right) \\
\sum_{l=1}^{L} X_{i j l t} \geq Q_{i j t}, i=\overline{1, m} ; j=\overline{1, n} ; \overline{\min } \overline{1, T} \\
\sum_{i=1}^{m} \sum_{j=1}^{n} \sum_{t=1}^{T} X_{i j l t} \geq \alpha_{1}, l=\overline{1, L} \\
X_{i j l t} \geq 0, i=\overline{1, m} ; j=\overline{1, n} ; t=\overline{1, T}
\end{gathered}
$$

where $\alpha_{j l t}, \beta_{j l t}-$ penalty multipliers. Problem (7) - (10) can also be formulated as a two-stage stochastic linear problem in the form:

$$
\begin{aligned}
& Z=\sum_{i=1}^{m} \sum_{j=1}^{n} \sum_{l=1}^{L} \sum_{t=1}^{T} R_{i l} X_{i j l t}+M y^{+}(\theta)+M y^{-}(\theta) \\
& \rightarrow \min , \\
& \sum_{l=1}^{L} X_{i j l t}-Y_{j l t}^{+}(\Theta) \leq P_{j l t}(\Theta), \\
& j=\overline{1, n} ; l=\overline{1, L} ; t=\overline{1, T} ; \\
& \sum_{i=1}^{m} U_{i j t} X_{i j l t}-Y_{j l t}^{-}(\Theta) \leq V_{j l t} P_{j l t}(\Theta), \\
& j=\overline{1, n} ; l=\overline{1, L} ; t=\overline{1, T} \text {; } \\
& \sum_{l=1}^{L} X_{i j l t} \geq Q_{i j t}, i=\overline{1, m} ; j=\overline{1, n} ; t=\overline{1, T}, \\
& \sum_{i=1}^{m} \sum_{j=1}^{n} \sum_{t=1}^{T} X_{i j l t} \geq \alpha_{1}, l=\overline{1, L} \\
& X_{i j l t} \geq 0, i=\overline{1, m} ; j=\overline{1, n} ; l=\overline{1, L} ; t=\overline{1, T}, \\
& y^{+}(\Theta)=\sum_{j=1}^{n} \sum_{l=1}^{L} \sum_{t=1}^{T} \alpha_{j l t} y_{j l t}^{+}(\Theta) \\
& y^{-}(\Theta)=\sum_{j=1}^{n} \sum_{l=1}^{L} \sum_{t=1}^{T} \beta_{j l t} y_{j l t}^{-}(\Theta) \\
& y_{j l t}^{+}(\Theta)=\max \left(0 ; \sum_{i=1}^{m} X_{i j l t}-P_{j l t}(\Theta)\right) \text {, } \\
& y_{j l t}^{-}(\Theta)=\max \left(0 ; \sum_{i=1}^{m} U_{i j t} X_{i j l t}-X_{j l t} P_{j l t}(\Theta)\right)
\end{aligned}
$$

$$
Z_{2}(X, \Theta)=\sum_{j=1}^{n} \sum_{l=1}^{L} \sum_{t=1}^{T} \beta_{j l t} \max \left\{0 ; \sum_{i=1}^{m} U_{i j t} X_{i j l t}-V_{j l t} P_{j l t}(\Theta)\right\}
$$

according to the rules for calculating subgradients

$$
\begin{gathered}
\hat{Z}_{1 x}(X, \Theta)= \begin{cases}a_{j l t}, & \text { if } \sum_{i=1}^{m} X_{i j l t} \geq P_{j l t}(\Theta) \\
0, & \text { otherwise }\end{cases} \\
\hat{Z}_{2 x}(X, \Theta)= \begin{cases}\beta_{j l t} U_{i j t}, & \text { if } \sum_{i=1}^{m} U_{i j t} X_{i j l t} \geq V_{j l t} P_{j l t}(\Theta) \\
0, & \text { otherwise }\end{cases}
\end{gathered}
$$


it follows that

$$
\begin{gathered}
\xi_{1 j}^{S}= \begin{cases}1, & \text { if } \sum_{i=1}^{m} X_{i j l t} \geq P_{j l t}(\Theta) \\
0, & \text { otherwise }\end{cases} \\
\xi_{2 j}^{S}= \begin{cases}U_{i j t}, & \text { if } \sum_{i=1}^{m} U_{i j t} X_{i j l t} \geq V_{j l t} P_{j l t}(\Theta) \\
0, & \text { otherwise }\end{cases}
\end{gathered}
$$

This means that the stochastic quasi-gradient of the objective function $Z(x, \Theta)$ of the problem (7) - (10) at any point can be determined by the formula of the following form:

$$
x i_{j}^{S}=\sum_{i=1}^{m} \sum_{l=1}^{L} R_{i l}-\sum_{j=1}^{n} \sum_{l=1}^{L} \sum_{t=1}^{T} \alpha_{j l t} \xi_{1 j}^{S}-\sum_{j=1}^{n} \sum_{l=1}^{L} \sum_{t=1}^{T} \beta_{j l t t} \xi_{2 j}^{S}
$$

where $\xi_{1 j}^{S}$ and $\xi_{2 j}^{S}$ determined according to (23) and (24).

Thus, according to the experimental calculation with the smallest distance of grain delivery, an economic prerequisite is created to reduce the cost of grain procurement in the grain processing industry.

Consider the model of transportation of grain in the integrated system "Field-preparation-processing".

Reducing transportation costs during transportation of grain in the integrated field-harvest-processing system, preserving their quality by accelerating transportation is achieved by attaching the most rational transportation patterns through optimal coordination and coordination of its component subsystems.

We have developed two-stage optimization models for managing the formation of stocks and the implementation of target batches of wheat in the production system "fieldprocurement-processing" $[1,3]$.

The mathematical model of grain transportation in the complex system "field-procurement-processing" is formulated as a production and transport problem of linear programming of large dimensionality.

It is necessary to find in the area of permissible values such solutions, at which the work performed by vehicles for the transportation of grain in this system is minimized.

We write the mathematical model in the form: minimize

$$
Z=\sum_{i=1}^{m} \sum_{l=1}^{L} \sum_{j=1}^{n} R_{i j l}^{(1)} X_{i j l}+\sum_{l=1}^{L} \sum_{K=1}^{K} \sum_{j=1}^{n} R_{l k j}^{(2)} Y_{l k j}
$$

under restrictions

on the volume of grain production by an unattached grain manufacturer

$$
\sum_{l=1}^{L} P_{l j}-\sum_{i=1}^{m} Q_{i j}=Q_{m+1, j}, j=\overline{1, n}
$$

if $Q_{m+1, j}<0$, then $Q_{m+1, j}=0$;

on the volume of short delivery of grain at the enterprise for the storage of grain

$$
P_{l j}-\sum_{i=1}^{m} X_{i j l}=H_{l j}, j=\overline{1, n} ; l=\overline{1, L}
$$

on the need for an unattached grain storage enterprise

$$
\sum_{i=1}^{m} Q_{j l}-\sum_{l=1}^{L} P_{l j}=P_{m+1, j}, j=\overline{1, n}
$$

if $P_{m+1, j}<0$, then $P_{m+1, j}=0$;

on the volume of grain to be sold on the free market

$$
Q_{i j}-\sum_{l=1}^{L} X_{i j l}=S_{l j}^{(1)}, j=\overline{1, n} ; i=\overline{1, m}
$$

For the volume of grain supply to unattached grain storage enterprises

$$
\sum_{K=1}^{K} F_{k j}-\sum_{l=1}^{L}\left(P_{l j}-H_{l j}\right)=A_{L+1, j}, j=\overline{1, n}
$$

if $A_{L+1, j}<0$, then $A_{L+1, j}=0$;

on the volume of short delivery of grain to the processing plant

$$
F_{k j}-\sum_{l=1}^{L} Y_{l k j}=B_{k j}, j=\overline{1, n} ; k=\overline{1, K}
$$

on the needs of an unattached grain processing plant

$$
\sum_{l=1}^{L}\left(P_{l j}-H_{l j}\right)-\sum_{k=1}^{K} F_{k j}=C_{k+1, j}, j=\overline{1, n}
$$

if $C_{k+1, j}<0$, then $C_{K+1, j}=0$;

for the volume of grain to be sold on the free market of a grain storage enterprise

$$
\left(P_{l j}-H_{l j}\right)-\sum_{k=1}^{K} Y_{l k j}=S_{l j}^{(2)}, j=\overline{1, n}, l=\overline{1, L}
$$

on non-negativity of variables

$$
\begin{gathered}
X_{i j l} \geq 0, Y_{l k j} \geq 0, \\
i=\overline{1, m} ; j=\overline{1, n} ; \\
l=\overline{1, L} ; k=\overline{1, K} ;
\end{gathered}
$$

where, $i$ - the index of grain producers (collective and state farms);

$j-$ the index of the batch of grain by purpose;

$l$ - the index of enterprises for the storage of grain (GRE);

$k$ - index of grain processing enterprises;

$Q_{i j}$ - the volume of production of the $j$-th batch of grain at the $i$-th manufacturer;

$P_{l i}$ - the volume of storage of the $j$-th batch of grain at the $l$-th enterprise for the storage of grain;

$R_{i l}^{(1)}$ - the distance from the $i$-th grain producer to the $l$-th enterprise grain storage;

$Q_{m+1, j}$ - the volume of production of the $j$-th batch of grain unattached by the grain producer;

$H_{l i}$ - the volume of short deliveries of the $j$-th batch of grain at the $l$-th enterprise for the storage of grain; 
$P_{m+1, j}$ is the storage volume of the $j$-th grain batch of an unattached grain storage enterprise at the $i$-th grain producer to be sold on the free market;

$S_{i j}^{(1)}-j$-th batch volume of grain from $i$-th grain producer to be sold on the free market;

$F_{k j}$ - the need of the $k$-th grain processing enterprise in the $j$-th batch of grain;

$R_{l k}^{(2)}$ - the distance from the $l$-th enterprise for the storage of grain to the $k$-th enterprise for processing;

$A_{l+1, j}$ - the volume of delivery of the $j$-th batch of grain of an unattached enterprise for the storage of grain;

$B_{k j}$ - the volume of short delivery of the $j$-th batch of grain to the $\mathrm{k}$-th grain processing enterprise;

$C_{k+1, j}$ - the need for an unattached grain processing enterprise in the $j$-th batch of grain;

$S_{l j}^{(2)}$ - the amount of $j$-th shipment of grain, subject to the implementation of free market enterprise $l$-th grain storage;

$X_{i j l}$ - volumes supplied $j$-th grain party $i$-th producer grain $l$-th grain storage entity;

$Y_{l k j}$ - volume supplied by $j$-th batch of grain from the $l$-th enterprise for grain storage $k$-th company for processing.

The task of transporting grain in the complex fieldharvesting-processing system was accomplished in two stages: a field-harvesting and a storage-processing, which determine the formation of optimal stocks and the implementation of target wheat lots.

When solving the problem in the system "fieldprocurement", the necessity was taken into account: timely delivery of products to their destinations with proper safety; effective use of vehicles, loading and unloading equipment of receiving and processing enterprises; rational use of technical capabilities of GRE for the operations of receiving, processing, placing the formation of batches of grain.

\section{Conclusion}

These solutions of the model (25) - (34) can be used to justify the capacity, throughput of the GRE and control the sale of grain. The developed models, in contrast to previously known ones, are built in accordance with the principles of targeting and taking into account the peculiarities of regional development. In the integrated fieldharvesting-processing system, on the example of the Ak- mola region, optimal options for attaching grain farms to grain receiving enterprises are determined on the basis of effective use of their technical ability in operations of receiving, processing, placing, forming batches of grain and the optimal scheme for linking grain receiving enterprises to processing plants taking into account the distance of transportation for the intended purpose, as well as the annual demand. These model solutions can be used to justify the capacity, throughput of grain receiving elevators and managing grain sales. The digital (dialogue) technology of optimization calculations "ASTYK", complex, implemented on the basis of standard software tools, can be used for a wide class of tasks for optimizing transport and logistics systems for grain processing in real time planning process.

\section{References}

[1] A. Saparbayev, Transport logistics in grain processing (Fortuna Polygraph LLP, 2019)

[2] A. Iztaev, T. Kulazhanov, A. Saparbayev, Innovative technologies and logistics of processing businesses in AIC (Fortuna Polygraph LLP, 2019)

[3] A. Bakaev, V. Revenko, V. Gritsenko, Information technology in transport, Vol. 3. Water transport (Naukova dumka, Kyiv, 2009)

[4] E. Vasilyeva, V. Levit, V. Livshits, Nonlinear transport problems on networks (Finansy i statistika, Moscow, 1981)

[5] L. Lesdon, Optimization of large systems (Nauka, Moscow, 1975)

[6] E. Krylatykh, A. Strokov, Food Security of Russia and the World: Theory and Methodology of Research, Analysis of Provision, Opportunities and Threats (Infra-M, 2019)

[7] A. Ganicheva, Mathematical methods and models in agroindustrial complex (Tver State Agricultural Academy, Tver, 2019)

[8] A. Makulova, Model assessment of agricultural production management (Fortuna Polygraph LLP, 2019)

[9] H.A. Taha, Operations Research: An Introduction, 10th edn. (Pearson, 2017)

[10] G.B. Dantzig, M.N. Thapa, Linear programming 2: theory and extensions (Springer Science \& Business Media, 2006) 